\title{
DEUX OBSERVATIONS D'ISOSPORA BELLI AU MAROC
}

\author{
Par C. CORCUFF
}

Isospora belli a été découverte en Orient, en 1915, par Wenyon et a été revue depuis par un certain nombre d'auteurs. Cette coccidie se rencontre en Orient avec un pourcentage variant de 0,4 à 4 . Elle a été observée en outre par Noc au Sénégal, par Brug à Java, par Haughwout et Schule aux Philippines, par Newham et Robertson en Afrique du Sud, par Castex et Greenway à Buenos-Aires, par C. Pinto, G. Pacheco et A. Prado au Brésil, par Wassell en Chine, par Pons en Indochine, par Connal et Young en Nigeria, par Franchini en Tripolitaine, par Chatridse et Kipschidse au Caucase et par Voskressenski en Azerbaidjan.

La présente note relate deux cas nouveaux observés au Maroc.

Observation I. - Es., de Rabat, nous est envoyé le 12 décembre 1931 , pour une recherche de l'amibe dysentérique, par le $\mathrm{D}^{\mathrm{r}}$ Guilmoto.

Le malade défèque au laboratoire et ses selles, molles, avec quelques glaires, sont portées immédiatement à la chambre de Foot. Le flocon muqueux que nous examinons d'abord ne présente pas d'amibes. Cependant quelques cristaux de Charcot-Leyden entraînés, avec une parcelle de matières, sous la lamelle de ce premier étalement, attirent notre attention et nous font persévérer. Notre examen reste négatif pour l'amibe dysentérique.

Nous reprenons un peu de selles, diluées dans du liquide physiologique, et nous trouvons facilement de nombreux oocystes de coccidie et de très nombreux cristaux de Charcot-Leyden.

L'examen poursuivi ne révèle la présence d'aucun autre parasite.

Nous interrogeons le malade sur son genre de nourriture et sur ses occupations. C'est un employé de l'Administration du Protectorat qui ne quitte son bureau que pour rentrer chez lui. Il n'élève ni poules, ni lapins, et nous affirme n'avoir pas mangé de lapin dans les jours qui précèdent. Il n'a pas de chien.

Nous le prions de vouloir bien revenir dans quelques jours, en lui recommandant de ne pas manger autre chose que de la viande de bœuf ou de veau et des légumes épluchés, jusqu'à sa prochaine visite, qui a lieu le 17 décembre, c'est-à-dire 5 jours après. Un nouvel examen est pratiqué dans les mêmes conditions que le précédent et nous trouvons exactement les mêmes oocystes de coccidie et les mêmes cris-

Annales de Parasitologie, $\mathrm{T}$. XIV; $\mathrm{N}^{\circ} 5 .-1^{\mathrm{er}}$ septembre 1936 , 
taux de Charcot-Leyden, en quantité sensiblement égale à la première observation (environ 2 oocystes, par champ $8 \times 6$, Leitz). Il n'y a également pas d'autres parasites; on ne trouve seulement que quelques rares leucocytes. Nous pratiquons immédiatement une coproculture (ac. chromique à $1 / 1000$ ).

Etant donné l'existence des seuls oocystes de coccidie, nous interrogeons le malade sur les signes cliniques qui ont déterminé sa visite au

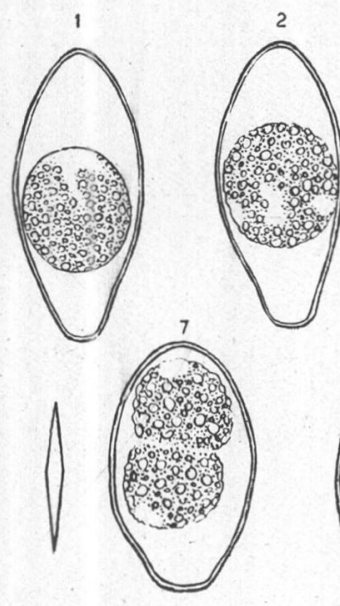

ఏ.

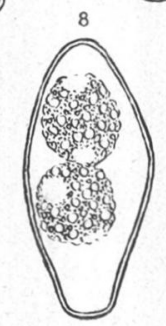

$\circ$

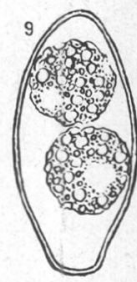

10

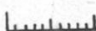

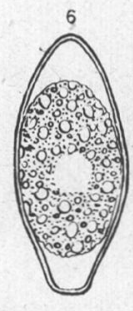

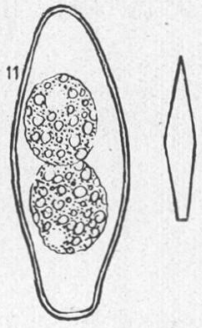

$50 \mu$

FiG, 1. - Isospora belli et cristaux de Charcot-Leyden. 1, 2, 3, 4, 5, 6, formes non segmentées (examen direct des selles du 17-12-1931); 7, 8, 9, 10, 11, formes segmentées, spores (coproculture le 19-12-1931).

médecin et nous apprenons que depuis plusieurs semaines ( 3 environ), « ça n'allait pas » ni au point de vue de l'état général, ni au point de vue intestinal. Le malade se fatiguait rapidement, maigrissait, perdait ses forces et transpirait facilement. Il n'avait jamais eu aussi froid le soir et la nuit que depuis ces derniers temps (il n'attachait d'ailleurs que peu d'intérêt à ce signe étant donné la saison). Température normale, à peine légère diminution de l'appétit. Depuis 8 jours environ, après les repas, le malade est pris de coliques assez violentes, siégeant assez bas et à droite, dans la fosse iliaque. A partir de ce moment-là, les selles sont devenues plus fréquentes, foncées, pâteuses, luisantes ; elles se succèdent dans la proportion de 5 à 6 par jour. Elles ne sont pas striées de sang; elles ne sont pas fétides. Il n’y a pas le « crachat rectal », 
Etat général assez bon, le malade continue son travail malgré la localisation douloureuse qu'il présente par moments.

Observation II. - G., de Petit-Jean, est conduit à notre laboratoire le 19 décembre 1931 par le $\mathrm{D}^{\mathrm{r}}$ Tissot lui-même pour une recherche d'amibes. Notre attention est attirée par la présence de cristaux de Charcot-Leyden. La selle qui nous est soumise est molle, brun verdâ, tre, épaisse, sans glaires, sans pus, très fumante (chaude). Nous ne trouvons pas de formes végétatives, ni de formes kystiques d'amibes, pas plus qu'aucun autre parasite, mais de temps en temps, nous rencontrons, avec quelques leucocytes, des oocystes de coccidie (1 tous les
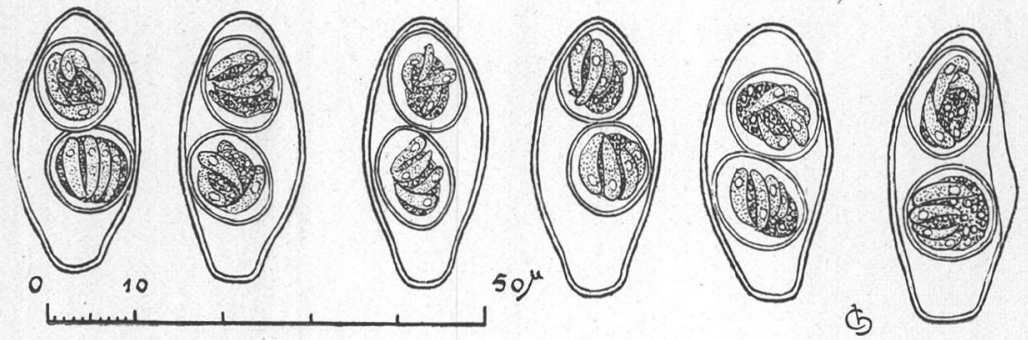

FIG. 2. - Isospora belli. Formation des sporozoïtes (coproculture le 20-12-1931).

5 à 6 champs $8 \times 6$ Leitz) de même forme et de mêmes dimensions que ceux observés dans le cas précédent $(25-33 \mu \times 12-16 \mu)$.

Notre interrogatoire porte immédiatement sur ce que le malade a pu ingérer. Nous éliminons toutes causes de parasitisme par un parasite égaré chez ce malade, colon de la région de Petit-Jean $(150 \mathrm{~km}$. de Rabat). Nous prions M. G., qui retourne chez lui le jour même, de bien vouloir observer une alimentation susceptible de ne pas apporter une cause d'erreur et de nous faire parvenir des selles aussi fraîches que possible dans quelques jours. Effectivement, le 23 décembre, nous recevons à 11 heures des selles émises à 6 heures du matin, dans lesquelles nous retrouvons nos oocystes dans les mêmes proportions. Coproculture immédiate venant compléter la coproculture faite avec la selle du 19.

M. G. présentait les signes cliniques suivants : son attention est attirée depuis quelque temps par l'apparition de selles anormales: molles, pâteuses, durant 4 à 5 jours, à raison de 3 ou 4 par jour, alternant avec des selles d'aspect sensiblement normal pendant deux jours environ. Les évacuations rapides de selles anormales correspondaient avec des sensations sourdes de coliques et des épreintes d'une certaine intensité, le tout dominé par un affaiblissement de l'état général. A l'examen 
clinique le $\mathrm{D}^{\mathrm{r}}$ Tissot a réveillé à la palpation de l'abdomen une douleur sur tout le trajet des colons (douleur en cadre). Température $37^{\circ} 5$, bon appétit, langue rose et humide.

Le rôle pathogène des coccidies intestinales de l'homme est encore imparfaitement connu. Dans les deux observations rapportées ici, la coccidiose ne s'est manifestée que par un petit nombre de selles en 24 heures, par des coliques plus ou moins intenses et par un affaiblissement de l'état général.

Institut d'Hygiène de Casablanca (Maroc). 